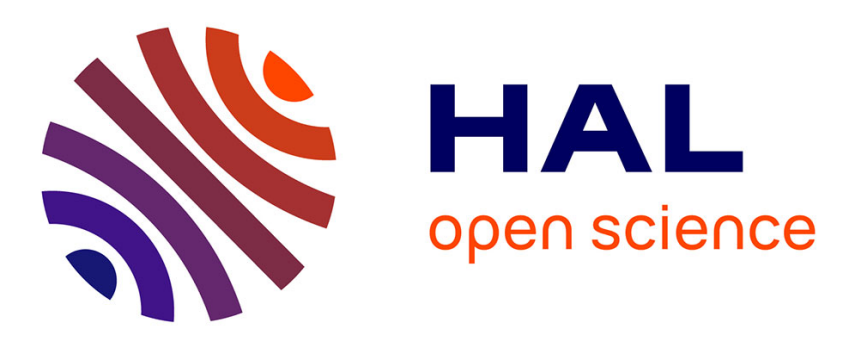

\title{
Thin structure filtering framework with non-local means, Gaussian derivatives and spatially-variant mathematical morphology
}

Tuan-Anh Nguyen, Alice Dufour, Olena Tankyevych, Amir Nakib, Eric Petit, Hugues Talbot, Nicolas Passat

\section{- To cite this version:}

Tuan-Anh Nguyen, Alice Dufour, Olena Tankyevych, Amir Nakib, Eric Petit, et al.. Thin structure filtering framework with non-local means, Gaussian derivatives and spatially-variant mathematical morphology. International Conference on Image Processing (ICIP), 2013, Melbourne, Australia. pp.1237-1241, 10.1109/ICIP.2013.6738255 . hal-01719128

\section{HAL Id: hal-01719128 \\ https://hal.univ-reims.fr/hal-01719128}

Submitted on 28 Feb 2018

HAL is a multi-disciplinary open access archive for the deposit and dissemination of scientific research documents, whether they are published or not. The documents may come from teaching and research institutions in France or abroad, or from public or private research centers.
L'archive ouverte pluridisciplinaire HAL, est destinée au dépôt et à la diffusion de documents scientifiques de niveau recherche, publiés ou non, émanant des établissements d'enseignement et de recherche français ou étrangers, des laboratoires publics ou privés. 


\title{
THIN STRUCTURE FILTERING FRAMEWORK WITH NON-LOCAL MEANS, GAUSSIAN DERIVATIVES AND SPATIALLY-VARIANT MATHEMATICAL MORPHOLOGY
}

\author{
T.A. Nguyen ${ }^{1}$ \\ A. Dufour ${ }^{2}$ \\ O. Tankyevych, A. Nakib, E. Petit ${ }^{1}$ \\ (1) Université Paris-Est, LISSI, Créteil, France \\ (2) Université de Strasbourg, ICube, France \\ (3) Université Paris-Est, LIGM, ESIEE-Paris, France \\ (4) Université de Reims, CReSTIC, France
}

H. Talbot ${ }^{3}$

N. Passat ${ }^{4}$

\begin{abstract}
Thin structure filtering is an important preprocessing task for the analysis of 2D and 3D bio-medical images in various contexts. We propose a filtering framework that relies on three approaches that are distinct and infrequently used together: linear, non-linear and non-local. This strategy, based on recent progress both in algorithmic/computational and methodological points of view, provides results that benefit from the advantages of each approach, while reducing their respective weaknesses. Its relevance is demonstrated by validations on $2 \mathrm{D}$ and $3 \mathrm{D}$ images.
\end{abstract}

Index Terms - Thin object filtering, mathematical morphology, Hessian filtering, non-local means, angiography.

\section{INTRODUCTION}

Thin patterns corresponding to anatomical (e.g., vessels, fibers, etc.) or artificial (e.g., catheters, etc.) structures are present in various $2 \mathrm{D}$ and $3 \mathrm{D}$ bio-medical images. Due to their geometric and size properties, such structures are hard to process, in particular because of noise -hiding them among non-relevant signal- and artifacts or partial volume effects, that tend to decrease their signal. This justifies the importance of thin structure filtering as a preprocessing task before the analysis of medical images in various contexts.

Several categories of filtering approaches have been developed and applied for medical image (pre)processing, in particular for thin structures analysis. Among them, three kinds of filters deserve our attention: $(i)$ non-local means (NLM) filters; ( $i i$ ) Gaussian derivative filters; and ( $i i i)$ spatially-variant mathematical morphology (SVMM) filters.

Non-local means filters [1] take advantage of the redundancy of information within an image. They act like averaging filters by considering weights according to the similarity of small windows around the regions of interest. These make them effective at removing noise while preserving local

This research work has received funding from the French Agence $\mathrm{Na}$ tionale de la Recherche (Grant Agreement ANR-2012-MONU-XXXX). structures. However, their drawback is their high computational cost, induced by the conjugate effects of the kernel and computation window sizes. NLM have been shown useful for curvilinear structures [2].

First- and second-derivative filters have been intensively considered for thin structures analysis. They generally combine a Gaussian (linear) scale space approach, with a Hessian matrix analysis [3]. A multi-scale framework can not only characterize thin structures, but also obtain information related to size and orientation. Hessian-based filters have been used in anisotropic diffusion schemes [4], to suppress noise while enhancing thin structures.

Mathematical morphology operators are well suited for thin structure enhancement since geometry can be directly considered via appropriate structuring elements. These include segments and paths, combined over families. These operators can then act as filters, for noise removal [5], but also for reconnection [6,7], even in the presence of heavy noise. In order to deal with the combinatorial issues related to the use of families of structuring elements, SVMM approaches have been developed [8].

These three families of filtering approaches present complementary strengths against one another's weaknesses, in terms of behaviour (denoising $v s$. reconnection), underlying strategy (linear vs. non-linear; local vs. non-local) and computational cost. In previous works [9] an hybrid approach involving Hessian-based and SVMM filtering was developed, mixing linear and non-linear approaches. This method essentially acted as an extensive filter that could reconnect vessels for segmentation purposes [10], but could not actually remove noise.

An other improvement of the method is that reconnection is only applied at the end of each segment, which makes it more efficient and less susceptible to changing the profile of the whole structure.

We now investigate the relevance of mixing local and nonlocal approaches. To this end, we introduce a filtering framework based on the three above approaches. Our purpose is threefold: $(i)$ filtering out the noise without altering the thin 
structures, $(i i)$ correcting the disconnections of such structures, while $($ iii) maintaining a low computational cost.

This article is organised as follows. Sec. 2 describes the combined filtering strategy. Sec. 3 proposes experimental validations on synthetic images. Sec. 4 shows results on real images, recall the contributions and outlines further works.

\section{METHODS}

In this section, we propose a combined filtering framework devoted to curvilinear objects. It especially aims at filtering out the noise and enhancing the smallest (low-intensity) vessels, and correctly dealing with disconnections.

The method takes as input a 2D or 3D grey-level image $I_{\text {in }}: E \rightarrow V$. The process is divided into four main steps: 1) an optimised NLM filter is applied to denoise the image while preserving the contrast and edges. 2) The Hessian matrix is then computed at different scales, resulting in a vesselness image and leading to define a vector image corresponding to the principal vessel directions. 3) This direction field is sampled at the endpoints of disconnected objects and dilated in order to produce dense and regular direction fields across disconnection gaps. 4) Spatially-variant morphological closing operation performs the final reconnection step.

The method finally provides as output a grey-level filtered image $\mathcal{F}\left(I_{i n}\right): E \rightarrow V$ of the input image $I_{i n}$, such that $I_{\text {in }} \leq \mathcal{F}\left(I_{\text {in }}\right)$ (i.e., $I_{\text {in }}(x) \leq \mathcal{F}\left(I_{\text {in }}\right)(x)$ for any $x \in E$ ), and enabling in particular to reconnect relevant high intensity line-like structures of $I_{i n}$.

\subsection{Step 1: Fast Enhanced NL-means}

In this step, the Fast Enhanced NLM filter is applied to $I_{i n}=$ $I$ to denoise the image while preserving the contrast and edges and resulting in image $I_{f}$. The NLM algorithm [1] is based on the natural redundancy of information in images. The restored intensity of the pixel $x, \operatorname{NLM}(I)(x)$ is a weighted average of all voxel intensities in image $I$ :

$$
I_{f}(x)=\operatorname{NLM}(I)(x)=\sum_{y \in E} w(x, y) I(y),
$$

where $w(x, y)$ is the weight assigned to $I(y)$ in the restoration of pixel $x$. The weight quantifies the similarity of pixels $x$ and $y$ and is normalized such that $w(x, y) \in[0,1]$ and $\sum_{y \in E} w(x, y)=1$. For each pixel $y$, the averaged Euclidean Distance (ED) is computed between $I\left(N_{y}\right)$ and $I\left(N_{x}\right)$. This classical ED is convolved with a Gaussian kernel, and is a measure of the distortion between pixel neighbourhood intensities. A drawback of this filter is its computing efficiency and the complexity of parameter fitting. Here, our enhancements improve both. We fit the weights automatically, inspired by Coupé et al. [2]. Specifically, to make the filter independent of neighbourhood size and to reduce the computational time, we normalize the classical ED by the number of elements. Then, the expression of the weights becomes:

$$
w(x, y)=\frac{1}{Z_{x}} \exp \left(-\frac{\sum_{p=1}^{\left|N_{x}\right|}\left(I^{(p)}\left(N_{x}\right)-I^{(p)}\left(N_{y}\right)\right)^{2}}{2 \beta \hat{\sigma}^{2}\left|N_{x}\right|}\right),
$$

where $I^{(p)}\left(N_{x}\right)$ is the value of the $p$ 's element of neighborhood $N_{x}$ in $I$. Now only the adjusting constant $\beta$ needs to be manually tuned, and $Z_{x}$ is a normalization constant. In the Gaussian noise case, $\beta$ is theoretically close to 1 if the estimation $\hat{\sigma}$ of the standard deviation of the noise is accurate. It is possible to reduce the computation time further by ignoring the small weight taking into account the mean and variance in the neighbourhood. To further improve efficiency, we grouped neighbourhoods to compute the weight when $x$ and $y$ are centred on two homogeneous blocks. Finally, our implementation takes advantage of a hybrid platform: MPI multi-threading on multi-cores machine with GPU acceleration.

\subsection{Step 2: vessel detection}

In this step, the Hessian matrix of $I_{f}$ is computed at different scales, resulting into a vesselness image $I_{\text {ves }}$ and leading to define a vector field image corresponding to the principal vessel directions: $I_{x_{1}}, I_{x_{2}}$ and $I_{x_{3}}$ (the latter in 3D only).

Given a set of different scales $\mathcal{S}$ to characterize vessels with different radii, the image $I_{f}$ is first convolved with a Gaussian kernel $G(x, s)=\left(2 \pi s^{2}\right)^{-N / 2} \cdot \exp \left(-\frac{|x|^{2}}{2 s^{2}}\right)$ at each scale $s \in \mathcal{S}$. At each point $x \in E$, its Hessian matrix $\mathcal{H}_{s}$ is then computed. After the eigen analysis of $\mathcal{H}_{s}$, the principal values are ordered (assuming bright objects on dark background): $\left|\lambda_{1}\right| \leq\left|\lambda_{2}\right| \leq\left|\lambda_{3}\right|$. The associated basis vectors $\left(\mathbf{e}_{1}, \mathbf{e}_{2}, \mathbf{e}_{3}\right)$ define the local orientation of the characterized shape in $x$. In particular, the vectors $\mathbf{e}_{\mathbf{1}}$ are stored in three images as the principal directions (along the principal axes): $I_{x_{1}}, I_{x_{2}}, I_{x_{3}}: E \rightarrow[-1,1]$.

Using the eigenvalues $\lambda_{1}, \lambda_{2}, \lambda_{3}$, a vesselness measure introduced by Frangi et al. [3] identifies vessels, appearing as bright objects on a dark background, and for 3D images can be expressed as :

$$
\nu(x, s)=\left\{\begin{array}{l}
0 \text { if } \lambda_{2}>0 \text { or } \lambda_{3}>0, \\
\left(1-e^{\frac{-R_{A}^{2}}{2 \alpha^{2}}}\right) \cdot e^{-\frac{R_{B}^{2}}{2 \beta^{2}}} \cdot\left(1-e^{\frac{-S^{2}}{2 \gamma^{2}}}\right) \text { otherwise }
\end{array}\right.
$$

with

$$
R_{A}=\frac{\left|\lambda_{2}\right|}{\left|\lambda_{3}\right|}, \quad R_{B}=\frac{\left|\lambda_{1}\right|}{\sqrt{\left|\lambda_{2} \lambda_{3}\right|}}, \quad S=\sqrt{\sum_{j=1}^{3} \lambda_{j}^{2}},
$$

in which $R_{A}$ differentiates between planar and line-like objects, $R_{B}$ describes blobs, and $S$ accounts for the intensity difference between objects and background. Parameters $\alpha, \beta$ 
and $\gamma$ influence the sensitivity of the filter to corresponding measures. The final vesselness result among the scales of $\mathcal{S}$ is chosen for each point $x$ as its maximal response $\nu_{\max }(x)=$ $\max _{s \in \mathcal{S}}\{\nu(x, s)\}$.

\subsection{Step 3: directional field correction}

In this step, from a thresholded version of $I_{\text {ves }}$, the direction images $I_{x_{1}}, I_{x_{2}}, I_{x_{3}}$, and a discrete skeleton, dense and regular vessel direction fields $I_{x_{1}}^{d}, I_{x_{2}}^{d}$ and $I_{x_{3}}^{d}$ are obtained for object reconnections.

In order to propagate objects outside their own boundary, we propose using a spatially-variant morphological closing [11], that requires a direction vector field extending beyond these boundaries. In our case, the directional information is necessary only as far as the dilation can reach. However, simply diffusing the directions obtained by second-order derivatives is not sufficient. Indeed, directions are typically unreliable at the end of tubular object segments. To solve this, we regularize the direction vector field. For this, the vesselness image $I_{\text {ves }}$ is thresholded so that most of the vessellike objects are preserved and results in an image $I_{v e s}^{t}$. Then, we obtain a morphological skeleton of $I_{v e s}^{t}$, from which we extract the endpoints [12]. By identifying the endpoints of this skeleton [13], we ensure that we use the tubular objects as markers for direction field propagation and further reconnection only between disconnected objects. We perform a morphological dilation of this direction field $\left(I_{x_{1}}, I_{x_{2}}, I_{x_{3}}\right)$ guided by endpoints with a structuring element of fixed size. This results into extended and regularized direction fields $I_{x_{1}}^{d}$, $I_{x_{2}}^{d}, I_{x_{3}}^{d}$.

\subsection{Step 4: vessel reconnection}

In this last step, a SV morphological closing operation is performed over the image $I_{f}$ with the aim of reconnecting vessels. First, a morphological dilation is applied with a structuring function $B: E \rightarrow 2^{E}$, providing, for each $x \in E$, a structuring element $B(x)$ centred on $x$, of fixed length, and oriented according to $\mathbf{e}_{\mathbf{1}}(x)$. The (discrete) direction $\mathbf{e}_{\mathbf{1}}$ of $B(x)$ is estimated from the images of regularized direction fields, $I_{x_{1}}^{d}, I_{x_{2}}^{d}$ and $I_{x_{3}}^{d}$, by defining a discrete segment.

This dilation, resulting in the image $\delta_{B}\left(I_{f}\right)$, is followed by an efficient adjunct erosion $\varepsilon_{B}$ [9]. Both computations $\left(\delta_{B}\right.$ and $\left.\varepsilon_{B}\right)$, then provide the final filtering result $\mathcal{F}\left(I_{f}\right)=$ $\varphi_{B}\left(I_{f}\right)$ with a low algorithmic cost. This efficient adjunction ensures that our filter is indeed a morphological closing.

\section{EXPERIMENTS}

In order to evaluate its effectiveness, the proposed filter was compared to the classical anisotropic diffusion filter [4]. To assess the relevance of mixing local and non-local approches,

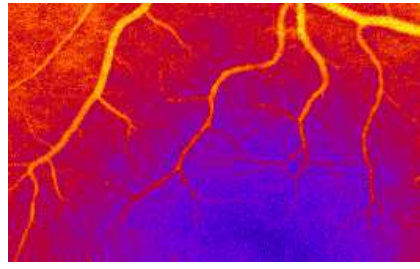

(a)

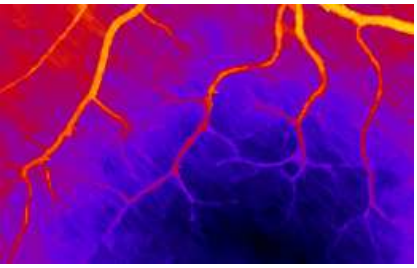

(b)

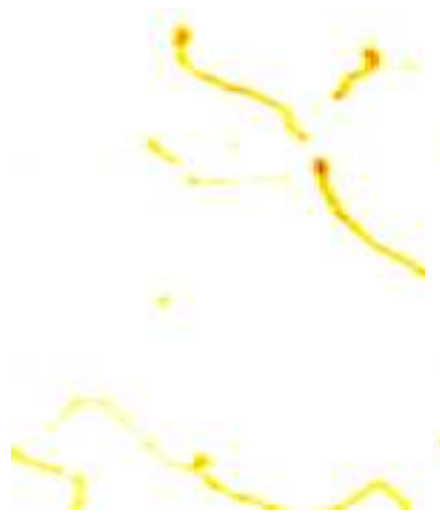

(c)

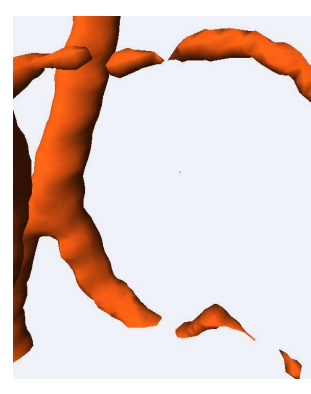

(e)

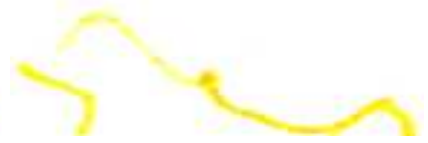

(d)

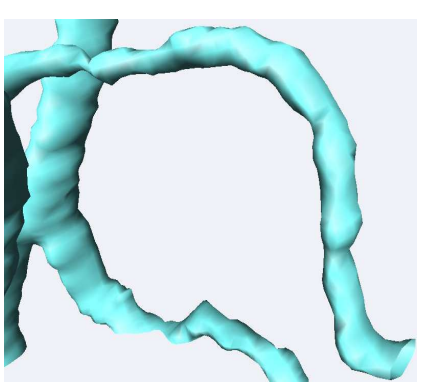

(f)
Fig. 2. (a,b) 2D fundus angiography images. (c,d) 2D neurite images. (e,f) 3D magnetic resonance angiography (isosurface). (a,c,e) Original images. (b,d,f) Filtered images.

it has also been compared with its components, namely, the NLM filter [1], and the morpho-Hessian filter [9] (see Fig. 1).

We used the synthetic dataset proposed in [14] for thin objects filtering validations. In addition to the disconnections induced by the low diameter at the convergence of the three branches, supplementary disconnections were introduced by decreasing the signal to noise ratio in each branch (Fig. 1(a)). Several noise levels have been tested, corresponding to the expected SNR in MR, CT or US data, but also to explore the limits of the filter in the worst cases, which do sometimes happen in clinical applications (Fig. 1(b-e)).

First, one can remark that the proposed filter has an invariant behaviour w.r.t. the SNR of the images. This leads to satisfactory ROC curves, which are not degraded for high levels of noise, by opposition to the morpho-Hessian and anisotropic 


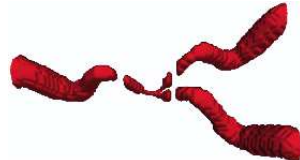

(a)

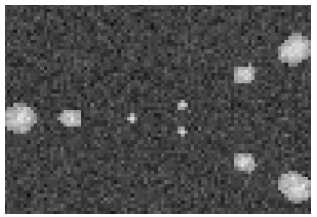

(b) $\sigma=10$

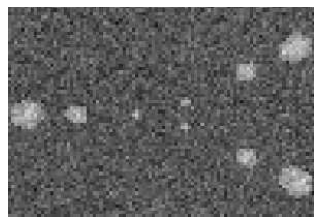

(c) $\sigma=20$

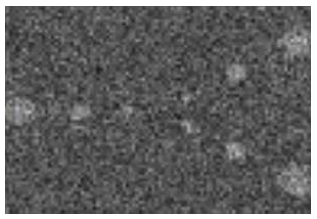

(d) $\sigma=40$

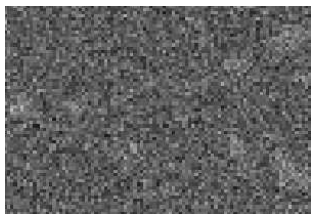

(e) $\sigma=80$

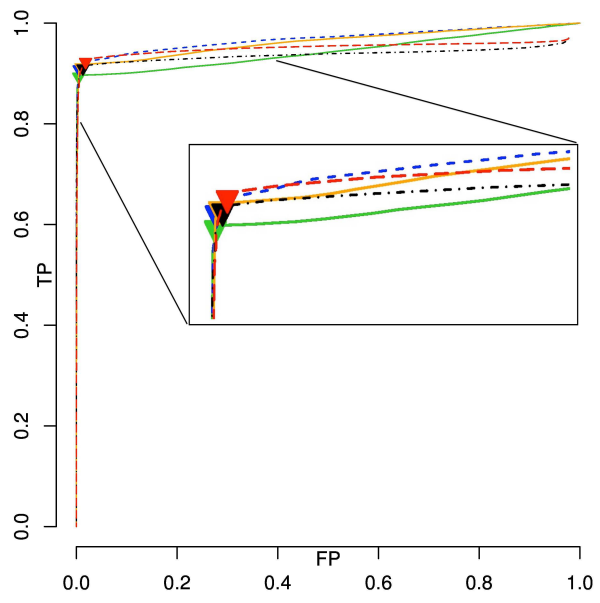

(f) $\sigma=10$

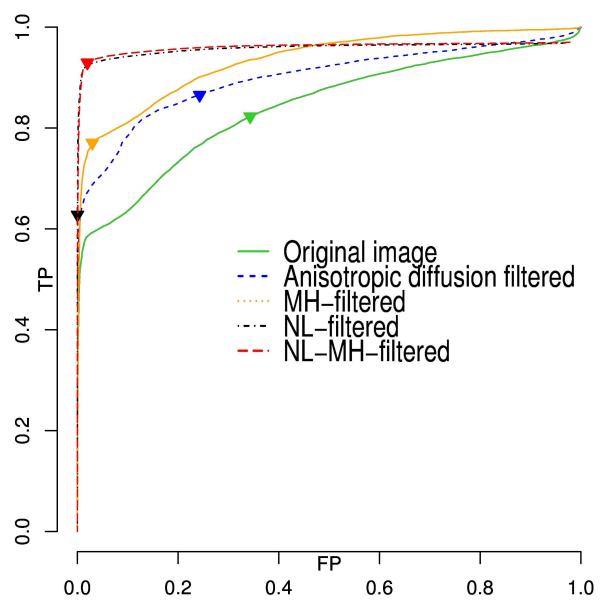

(h) $\sigma=40$

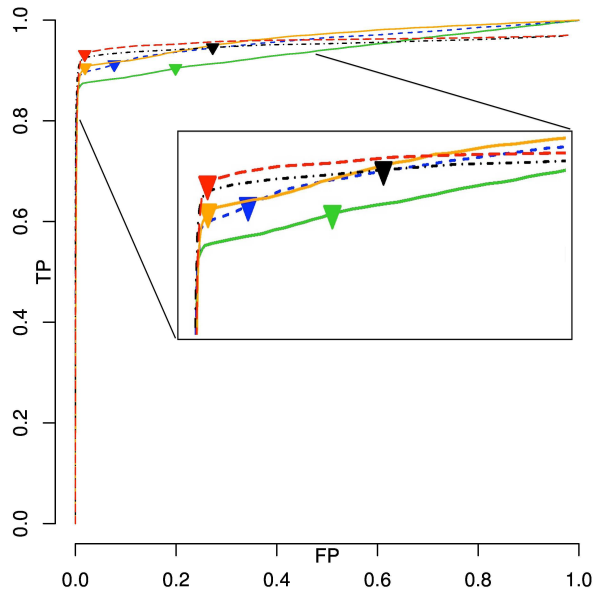

(g) $\sigma=20$

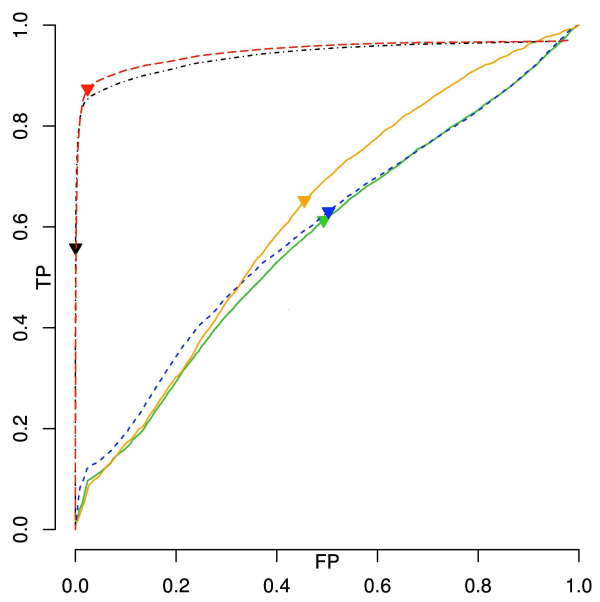

(i) $\sigma=80$

Fig. 1. (a) The phantom used for validations. (b-e) Slices of the associated images for various levels of noise (see text). (f-i) ROC curves of thresholding operations performed on the original/filtered image with different levels of noise (see text). The triangular dot on each curve indicates when a correct reconnection of the segmented structure has been obtained.

diffusion filters. This quality is shared with the NLM filter.

Nevertheless, if both filters present similar results from a quantitative point of view, the one we propose is superior to the NLM filter alone from a qualitative point of view. Indeed, the reconnection point is always obtained closer to the $(0,1)$ point of the ROC diagram. In other words, the structures that are reconnected are both relevant and non-noisy ones. In contrast, by itself the NLM filter often reconnects either structures that are non-complete, or strongly noisy.

\section{RESULTS AND CONCLUSION}

The proposed filtering method was applied to several families of 2D and 3D (bio)medical images, including 2D fundus angiography data, 2D neurite images and 3D angiography MR images. Fig. 2 shows some sample results, that confirm the experimental validations of Sec. 3. In particular, we observe that some of the gaps have been successfully corrected without introducing significant artifacts. As well, noise is reduced, contrast is enhanced and thin structures are preserved.

Nevertheless, some other disconnections -namely those presenting a large gap between two segments- may not be corrected by this filter. To deal with this issue, the SVMM part of the method could be improved by using path-based structuring elements [15]. Moreover, in order to propose a more complete family of useful filters for thin and tubular structures, future works will consider not only extensive operators, but also anti-extensive ones, that will aim to repair erroneous connexions between segments. 


\section{REFERENCES}

[1] A. Buades, B. Coll, and J.-M. Morel, "A review of image denoising algorithms, with a new one," Multiscale Model Sim, vol. 4, pp. 490-530, 2005.

[2] P. Coupé, P. Yger, S. Prima, P. Hellier, C. Kervrann, and C. Barillot, "An optimized blockwise nonlocal means denoising filter for 3-D magnetic resonance images," IEEE T Med Imaging, vol. 27, pp. 425-441, 2008.

[3] A. F. Frangi, W. J. Niessen, R. M. Hoogeveen, T. van Walsum, and M. A. Viergever, "Model-based quantitation of 3-D magnetic resonance angiographic images," IEEE T Med Imaging, vol. 18, pp. 946-956, 1999.

[4] R. Manniesing, M. A. Viergever, and W. J. Niessen, "Vessel enhancing diffusion: A scale space representation of vessel structures," Med Image Anal, vol. 10, pp. 815-825, 2006.

[5] M. H. F. Wilkinson and M. A. Westenberg, "Shape preserving filament enhancement filtering," in MICCAI. 2001, vol. 2208 of LNCS, pp. 770-777, Springer.

[6] P. Soille and H. Talbot, "Directional morphological filtering," IEEE T Pattern Mach Anal, vol. 23, pp. 13131329, 2001.

[7] C. L. L. Hendriks, "Constrained and dimensionalityindependent path openings," IEEE T Image Process, vol. 19, pp. 1587-1595, 2010.

[8] R. Verdú-Monedero and J. Angulo, "Spatially-variant directional mathematical morphology operators based on a diffused average squared gradient field," in ACIVS. 2008, vol. 5259 of LNCS, pp. 542-553, Springer.

[9] O. Tankyevych, H. Talbot, P. Dokládal, and N. Passat, "Direction-adaptive grey-level morphology. Application to 3D vascular brain imaging," in ICIP, 2009, pp. 22612264.

[10] A. Dufour, O. Tankyevych, B. Naegel, H. Talbot, C. Ronse, J. Baruthio, P. Dokládal, and N. Passat, "Filtering and segmentation of 3D angiographic data: Advances based on mathematical morphology," Med Image Anal, In Press.

[11] H.J.A.M. Heijmans, "Morphological image operators," in Advances in Electronics and Electron Physics Suppl., vol. 1. Boston: Academic Press, 1994.

[12] J. Chaussard and M. Couprie, "Surface thinning in 3D cubical complexes," in IWCIA. 2009, vol. 5852 of LNCS, pp. 135-148, Springer.
[13] G. Bertrand and G. Malandain, "A new characterization of three-dimensional simple points," Pattern Recogn Lett, vol. 15, pp. 169-175, 1994.

[14] S. R. Aylward and E. Bullitt, "Initialization, noise, singularities, and scale in height ridge traversal for tubular object centerline extraction," IEEE T Med Imaging, vol. 21, pp. 61-75, 2002.

[15] F. Cokelaer, H. Talbot, and J. Chanussot, "Efficient robust d-dimensional path operators," Selected Topics in Signal Processing, IEEE Journal of, vol. 6, no. 7, pp. 830-839, 2012. 